\title{
Early Return Of Activity Of Daily Living After Surgery Of Pfna Li Fixation In Elderly Osteoporotic Intertrochanteric
}

\author{
Mohammed Hazim M.Nouri (F.I.C.M.S.Otho.) *, Bilal Salman W. Al-Rawi (C.A.B.M.S Ortho)**
}

\begin{abstract}
Background: Hip fractures are common, morbid, and costly health events that threaten independence and function of older patients. Early functional recovery is extremely important for elderly patients with femoral intertrochanteric fractures to shorten their hospital stay, omit the necessity of the further nursing and care services, and reduce associated medical expenditures. Assessment of (ADL) is an important predictor of outcomes Of orthopedic surgery in very early postoperative period at two weeks and a month postoperatively using Japanese orthopedic score get a quick recovery of ADL \& return the patient to his or her premorbid level function reducing the morbidity and mortality.

Objectives: We evaluate in our study the Return of ADL function in 27 cases of elderly with intertrochanteric hip fractures treated with closed reduction and surgical fixation with proximal femoral nail anti rotation in very early postoperative period at two weeks and a month postoperatively.

Type of the study : Prospective observational stud
\end{abstract}

Methods: Prospective observational study of 27 consecutively patients with an intertrochanteric fracture
Stable and unstable fractures treated between December 2015 and Augest 2016 were enrolled in study.

Results: Regain in a walking ability and daily living activities which indicate a fast recovery in early days and weeks after surgery in contrary the regular fellow up a month up to 3 month revealed a slower recovery from that achieved in early weeks

Conclusions: Use of proximal femoral nailing in intertrochanteric elderly osteoporostic fracture has a significant impact on early functional recovery

Keywords: Pfna Elderly Osteoporotic Intertrochanteric Fracture

\section{Al-Kindy College Medical Journal 2017: Vol.13 No.1 Page: 46-50}

$$
\begin{aligned}
& \text { *Mohammed Hazim M.NOURI (F.I.C.M.S.Otho.), } \\
& \text { **Bilal Salman W. Al-Rawi (C.A.B.M.S Ortho) }
\end{aligned}
$$

Received $15^{\text {th }}$ Feb 2017, accepted in final $30^{\text {th }}$ March 2017 Corresponding to Bilal Salman W. Al-Rawi (C.A.B.M.S Ortho)
$\mathrm{H}$ ip fractures are a serious and common consequence of orthopedic trauma in elderly patients. Worldwide hip fracture rates are expected to increase to approximately 21 million per year by $2050(1)$. In the United States, the incidence of hip fractures is 309,500 per year. Healthcare system adaptations are necessary to accommodate such a large number of patients. Hip fracture prognosis is quite poor, with one-year mortality rates estimated to range from $20 \%$ to $30 \%(2)$. Among those patients who were independent prior to fracture, one year after hip fracture, $25 \%$ remained in nursing homes, and $60 \%$ required assistance in one or more activities of daily living. With such critical consequences, much research is being conducted to improve these outcomes. Older adults are typically high-risk candidates for surgery due to several factors. Many are afflicted with serious co-morbidities. A large proportion has pre-existing functional deficits that not only contribute to sustaining a fall, but also limit recovery after surgery. Homeostenosis, a decreased ability to compensate and maintain homeostasis when the body is stressed, might contribute to complications that can arise when an elderly patient undergoes surgery. Some complications are specific to older patients, and can result in geriatric syndromes associated with poor survival (3). In hip fracture patients, operative delay beyond $48 \mathrm{hr}$ after admission may increase the odds of 30-day all-cause mortality by $41 \%$ and of one-year all-cause mortality by $32 \%$. Potential residual confounding factors in observational studies may limit definitive conclusions. Although routine surgery within $48 \mathrm{hr}$ after admission is hard to achieve in most facilities, anesthesiologists must be aware that an undue delay may be harmful to hip fracture patients, especially those at relatively low risk or those who are young (4) .

According to AO/OTA alphanumeric classification intertrochanteric fractures (Type 31A) Bone $=$ femur $=$ 3, Segment $=$ proximal $=1$, Type $=A 1, A 2, A 3$.

A1: simple (two-part) fractures, with the typical oblique fracture line extending from the greater trochanter to the medial cortex; the lateral cortex of the greater trochanter remains intact.

A2: fractures are comminuted with a posteromedial fragment; the lateral cortex of the greater trochanter, however, remains intact. Fractures in this group are generally unstable, depending on the size of the medial fragment 
A3: fractures are those in which the fracture line extends across both the medial and lateral cortices; this group includes the reverse obliquity pattern or subtrochanteric extensions (5) .Japan is a country of longevity with women's average life expectancy exceeding 80 years of age. Today, the prevalence of osteoporosis in Japan is around 12 million and the hip fracture incidence rate in the oldest population (over 75) is increasing dramatically in both men and women. The Japanese Orthopaedic Association Hip Score is widely used in Japan, assess quality of life (QOL) and activity of daily living from the viewpoint of patients which was used in our study (6) WeadvocateArbeitsgemeinschaftfürOsteosynthesscoref ragen (AO)/Association for the Study of Internal Fixation (ASIF) principle of accurate anatomic reduction and stable internal fixation in the management of intertrochanteric fractures and apply this principle in the management of these cases in our practice. Based on previously reported high morbidity and managementrelated complications in patients with this fracture, various internal fixation devices and operative techniques have been developed to reduce management-associated complications. Currently, the most frequent devices used to manage this condition have been sliding compression hip screws with side plate assemblies including dynamic hip screw (DHS; Synthes, Paoli,PA, USA) and intramedullary fixation devices including proximal femur nail (PFN; Synthes) and gamma nail (GN;Stryker, Mahwah, NJ, USA). Although there have been controversies in the selection of the preferred fixation device, the intramedullary fixation devices have become increasingly popular due to the proven biomechanical and biological advantages . Numerous improved operative techniques with the newer intramedullary fixation devices have been introduced as well. That is, in an effort to reduce the incidence of intra- and/or postoperative complications, the 2 basic approaches that have been taken: design modification of the implant and modified reduction technique to achieve greater stability. The device must stabilize the fracture against deforming forces until union establishment. The fracture pattern, bone density and comorbidities in the patients were the uncontrollable factors in reducing the surgery-related complication rate. However, the surgery-related complications could be reduced by the advanced surgical management utilizing the new fixation devices and improved operative techniques, and consequently the hospitalization period also could be reduced (7).

Methods: Prospective observational study of 27 consecutively patients with an intertrochanteric fracture, There were 14 men and 13 women and mean age at time of fracture was 72.3+_9.64 years (range 58-89 years). Fracture classifications according to the $A O$ classification were: 6 cases of $31 \mathrm{~A} 1,17$ cases in $31 \mathrm{~A} 2$ and 4 cases in $31 \mathrm{~A} 3$ fractures. Stable fractures were of
$31 \mathrm{~A} 1 .$, and unstable fractures were considered of $31 \mathrm{~A} 2$ and 31A3 fractures treated between December 2015 and Augest 2016 were enrolled in study. The inclusion criteria were an age of $>55$ years with a community ambulatory with or without a cane before surgery. Patients with a pathologic fracture or severe medical comorbidities were excluded.
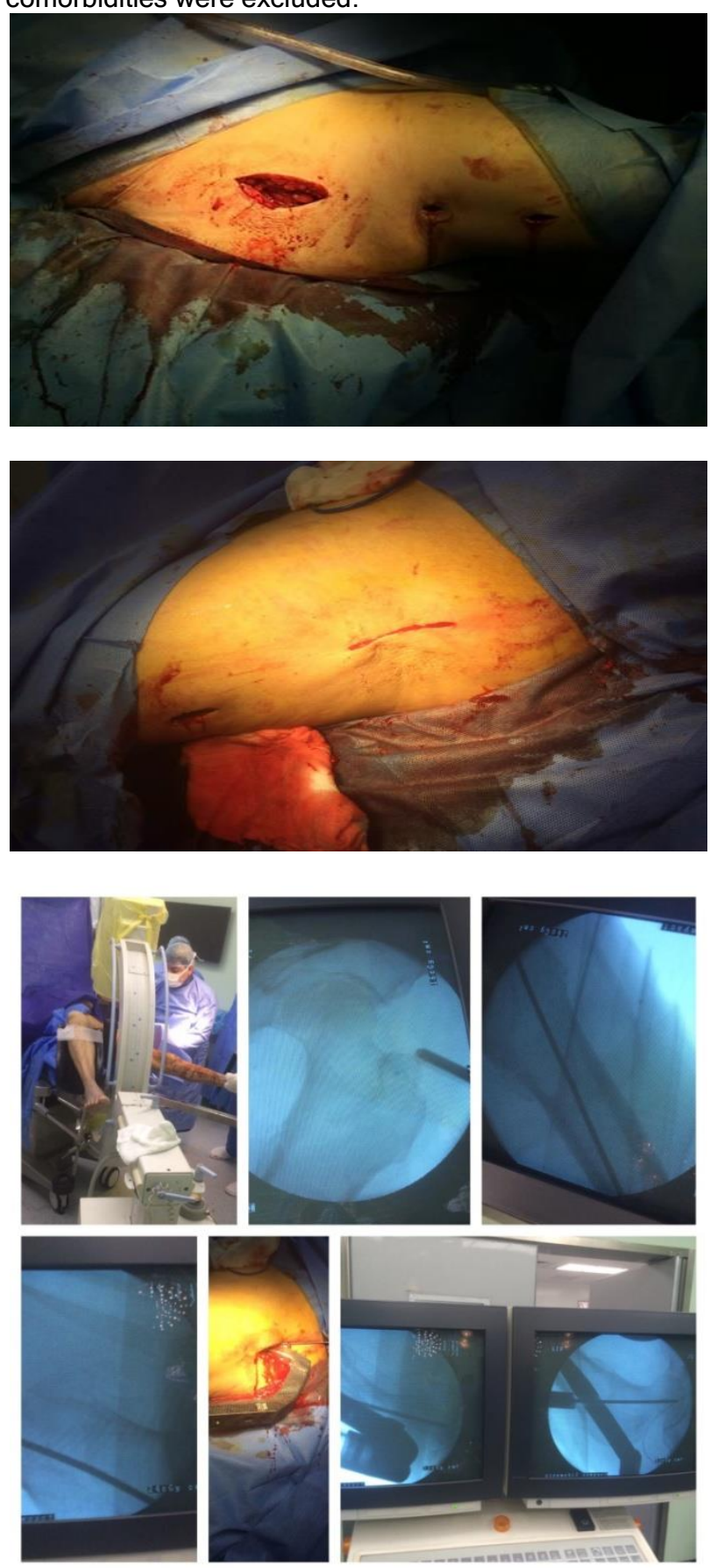

The patients were placed supine on a fracture table, closed reduction was confirmed by fluoroscopy before draping. The acceptable reduction was when neck-shaft angle was reduced within $<5^{\circ}$ and fracture site displacement $<4 \mathrm{~mm}$ as compared to normal side. A guide wire for the nail was placed to the tip of the greater trochanter and the position of the guide pin was checked 
fluoroscopically in both planes. A 17-mm-diameter double reamer was then inserted to cut a path for the nail and the PFNA nail was inserted. The guide wire for the large lag screw was targeted to be centrally located in antero posterior (AP) view and centrally or posteriorly located in lateral view, as determined using a C-arm image intensifier. The PFNA blade was then advanced to locate its tip as close to the subchondral bone (to a tip-apex distance (TAD) of $<20 \mathrm{~mm}$ ), which was 5-10 $\mathrm{mm}$ less than measured length, and the fracture was under compression with special blade compression system during unlocking of inserted blade device. Distal static locking screws were then inserted into the nail using the screw guide. All patients were given a single dose of prophylactic antibiotics with induction of anesthesia before surgery and low molecular weight heparin was administered postoperatively until the patients started to ambulate with assistance. After surgery, patients were instructed to stand with assistance with no weight bearing restriction and to use an assistive device (walker, crutches, or cane), as tolerated, on the 3rd postoperative day. As walking ability improved, assistive devices were changed at the discretion of responsible physical therapists. Patients were assessted in an early postoperative period at two weeks a month and 3 month postoperatively using Japannese orthopedic score evaluation regarding pain , walking activity and activities of daily livings were performed . Postoperative clinical outcome was evaluated using the recovery rates to prefracture status after surgery.

Results :All patients underwent closed reduction for intertrochanteric fracture. There were 14 men and 13 women figure (1) and mean age at time of fracture was $72.3+$ 9.64 years (range 58-89 years) figure (2), Waiting Period: $5.15 \pm 2.838$ preoperatively and In-patient Stay: $1.78 \pm 1.761$ postoperatively. Fracture classifications according to the AO classification were: 6 cases of $31 \mathrm{~A} 1,17$ cases in $31 \mathrm{~A} 2$ and 4 cases in $31 \mathrm{~A} 3$ fractures. Stable fractures were of $31 \mathrm{~A} 1$., and unstable fractures were considered of $31 \mathrm{~A} 2$ and31A3 fractures Figure (3) ,side of 16 left and 11 Right side figure (4). All patients underwent operative treatment after obtaining medical clearance for those they have comorbities Figure (5). Mean operative time (skin incision to wound closure) was $77.7 \mathrm{~min}$ (range $35-120 \mathrm{~min}$ ). Mean operation times were significantly dependent on fracture types $(P=$ $0.009)(48.9 \pm 29.0 \mathrm{~min}$ for stable fractures and $88.7 \pm$ $30.4 \mathrm{~min}$ for unstable fractures).
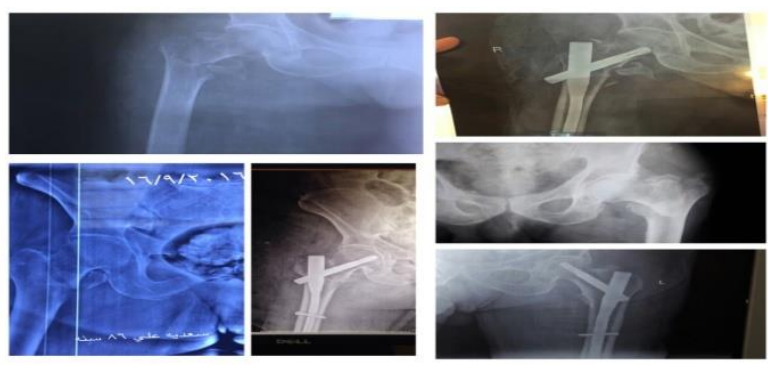

Statistical Analysis: The ANOVA ( F-test) and the kruskal-Willis test were used to analyze continuous data. Results were expressed as mean and standard deviation for pain ,walking ability and ADL table (1) ,figure (6). Statistical significance was accepted for $\mathrm{P}<$ 0.05 Table (2) .
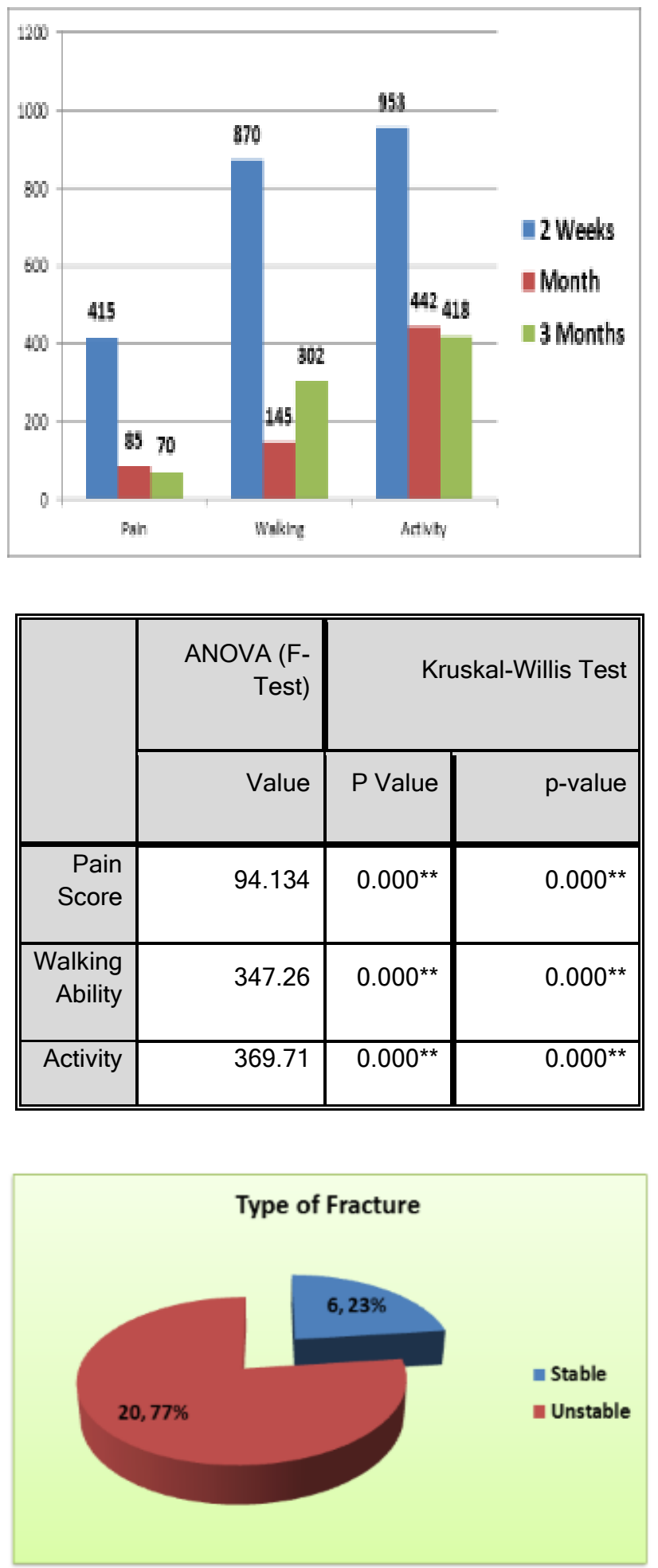
Discussion: Geriatric osteoporotic fractures is major challenging for surgeon decision and fracture fixation problem ,osteoporotic patients differ from normal subjects in bone mineral composition, bone mineral content and crystallinity ( poor quality and quantity ). so that we treat our 27 patients with trochanteric fracture stable and unstable types by closed reduction and proximal femoral nail instrumentation and we thought is considered a unique elderly osteoporotic fracture stable fixation and improve the outcome, reducing the surgical complication and postoperative morbidity and mortality which is currently world widely used and a device designed by AO/ASIF(proximal femoral nail antirotation). We know the Fracture healing is a complex process of bone regeneration, involving a well-orchestrated series of biological events that follow a definable temporal and spatial sequence that may be affected by both biological factors, such as age and osteoporosis, and mechanical factors such as stability of the osteosynthesis. Advantages of intramedullary nailing for hip fracture fixation include a more efficient weight bearing and load transfer due to the proximity of the implant to the medial calcar, less implant strain and shorter lever arm because of its closer positioning to the mechanical axis of the femur, significantly less soft tissue disruption and periosteal stripping of the femoral cortex, shorter operative time and hospital stay, fewer blood transfusions, better postoperative walking ability, and lower rates of leg-length discrepancy. Compromise of the posteromedial cortex and/or the lateral cortex, a subtrochanteric extension of the fracture, and a reversed obliquity fracture pattern represent signs of fracture instability, warranting the use of intramedullary nailing(8). We believe PFNA is anatomically designed guarantees an optimal fit in the femur, has a mediallateral angle of $6^{\circ}$ this allows insertion at the tip of the greater trochanter, Optimal stress distribution the flexible PFNA tip eases insertion and reduces stress on the bone at the tip of the PFNA. Rotational and angular stability achieved with one single element Large surface and increasing core diameter of pfna blade guarantee maximum compaction and optimal holdin bone Increased stability caused by bone compaction around the PFNA blade retarding rotation and varus collapse also the PFNA blade had a significantly higher cut-out resistance in comparison with commonly-used screw systems attributed to firstly Compaction of cancellous bone ( Inserting the PFNA blade compacts the cancellous bone) secondly the large core diameter of pfna blade providing additional anchoring, which is especially important in osteoporotic bone which is automatically locked to prevent rotation of the blade and femoral head. Static or dynamic locking can be performed via the aiming arm with PFNA standard.

Close look to our postoperative results immediately after surgery and two week after surgery. All patients were managed with a similar postoperative physiotherapy protocol, which consisted of early mobilization on the first day postoperatively by an experienced physiotherapist using a walker as assisting device and walking with weight-bearing as tolerated with good pain control and according to fracture type ,we find a large regain in a walking ability and daily living activities which indicate a fast recovery in early days and weeks after surgery in contrary the regular fellow up a month up to 3 month revealed a slower recovery from that achieved in early weeks which give us a very important points : a good pain control management, stability of the PFNA device security of fixation with acceptable close reduction parameters which was mention previously we can get our goal of earily mobility of elderly with hip fracture out of bed with assisting walking device decreasing morbidities postoperatively like pressure sore ,chest infection and DVT with there thromboembolic complications most of patient discharge from hospital on second day after surgery and during fellow up we record the total score at 3month postoperatively a hip score at 3 month of fair to good collectively, we believe it may attributed to mean age group in our study and to preexisting morbidities of our pateints which are consist with most of studies, Cooper C, Cummings S, Lyons A, et al in the assessing the crippling consequences of fractures and their impact on quality of life, $60 \%$ have difficulty with at least one essential activity of daily living, and $80 \%$ are restricted in other activities, such as driving and grocery shopping among those patients who were independent prior to fracture, one year after hip fracture, $25 \%$ remained in nursing homes, and $60 \%$ required assistance in one or more activities of daily living (9) We observe in our patients there slow improvement in pain in the period of days upto one month postoperative and we mange our patient with good analgesia which mostly at entry site of nail getting patient mild claudication. Regarding the mortality we have no mortality in our 27 patients in first 3 month of hip fracture in whom any impaired cognitive status, immobile patient and pathological fracture are excluded from our patients , limitation of our study due to the small number of patient sample and the short time of fellow up (3 month) . Tanja et al There were 192 hip fracture patients in total (59 $(30.7 \%)$ males and 133 (69.3\%) females). The mean age was 76.9 (age range from 65 to 91). Forty-seven $(35.6 \%)$ patients were older than 80 years of age. There were no deaths during surgery .Six months after admission for a hip fracture Six months after admission for a hip fracture 48 patients died, accounting for the sixmonth mortality rate $25 \%$., Cognitive functioning may be regarded as the most sensitive indicator of physiological ageing and poor morbidity pre-fracture status(10)

Conclusion: Use of proximal femoral nailing in intertrochanteric elderly osteoporostic fracture has a significant impact on early functional recovery. PFN is an intramedullary load-bearing device that allows for immediate postoperative weight bearing, with an antirotation screw allowing controlled impaction of the metaphyseal fracture zone. The PFN device reduced iatrogenic tissue trauma and reoperation rate. The present study showed that the PFN device can be used effectively to treat trochanteric fractures and may be the best choice particularly in unstable trochanteric fractures 
because of its low reoperation rate . Surgery-related complications could be reduced by the improved reduction and operative techniques, and consequently the hospitalization period also could be reduced.

\section{Reference}

1- Gullberg B, Johnell O, Kanis JA. World-wide projections for hip fracture.Osteoporosis International. 1997;7:407-13. [PubMed].

2- Keene GS, Parker MJ, Pryor GA. Mortality and morbidity after hip fractures. British Medical Journal. 1993;307:1248-60. [PMC free article] [PubMed].

3- Konstantin V. Grigoryan, MS, ${ }^{*}$ HoumanJavedan, $\mathrm{MD}, \dagger$ and James L. Rudolph MD, Ortho-Geriatric Care Models and Outcomes in Hip Fracture Patients: A Systematic Review and Meta-Analysis, 2014; id=23912859 pubmed .

4- Can J Anaesth. 2008 Mar Is Operative Delay Associated with Increased Mortality of Hip Fracture Patients? Systematic Review, Meta-Analysis, and MetaRegression

https://www.ncbi.nlm.nih.gov/pubmed/18310624.

5- AO/OTA Fracture and Dislocation Classification AOTrauma
https://aotrauma.aofoundation.org/.../classifications/.../A O-OTA-Fracture-and-Dislocation.

6- Sone T, et al. Prevalence of osteoporosis in Japan and the international comparison. Nippon Rinsho. 2004; 62 (Suppl.2):1972004 Japan - International Osteoporosis Foundation https://www.iofbonehealth.org/sites/default/files/.../Asian regional_audit.

7- The Stability Score of the Intramedullary Nailed Intertrochanteric Fractures: Stability of Nailed Fracture and Postoperative Patient Mobilization ;Published online February 20, 2013. https://www.ncbi.nlm.nih.gov/pmc/articles/PMC3582866/ 8- ) Parker MJ, Handoll HH.Gamma and other cephalocondylic intramedullary nails versus extramedullary implants for extracapsular hip fractures in adults. Cochrane Database Syst Rev. 2010(9):CD000093 .

9- Cooper C, Cummings S, Lyons A, et al. The crippling consequences of fractures and their impact on quality of life.American Journal of Medicine. 1997;103:12S-9S. [PubMed].

10- Factors Influencing The Six-Month Mortality Rate In Patients With A Hip Fracture ZdrVarst. 2016 Jun 1; 55(2): 112-117. Published online 2016 Feb 11. doi: 10.1515/sjph-2016-0015 ;PMCID: PMC4845770 